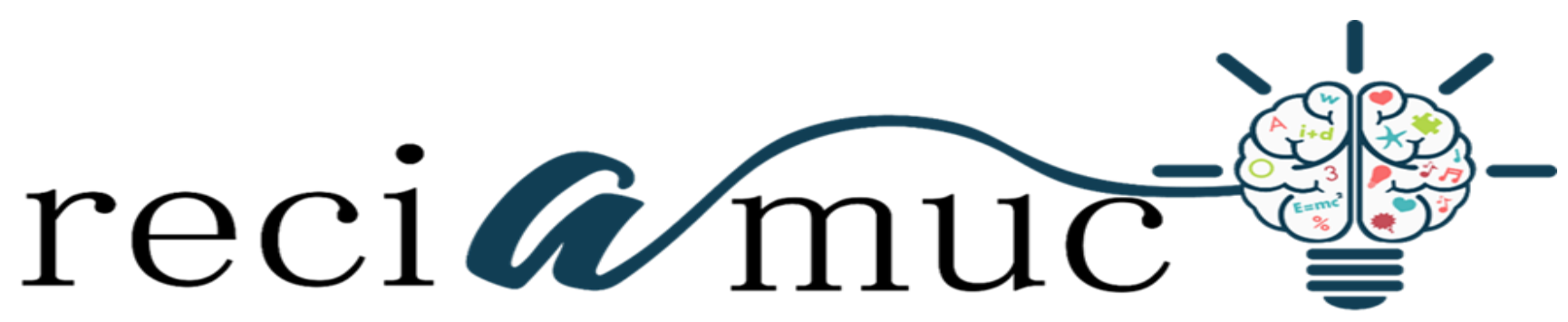

Revista científica de investigación actualización del mundo de las ciencias

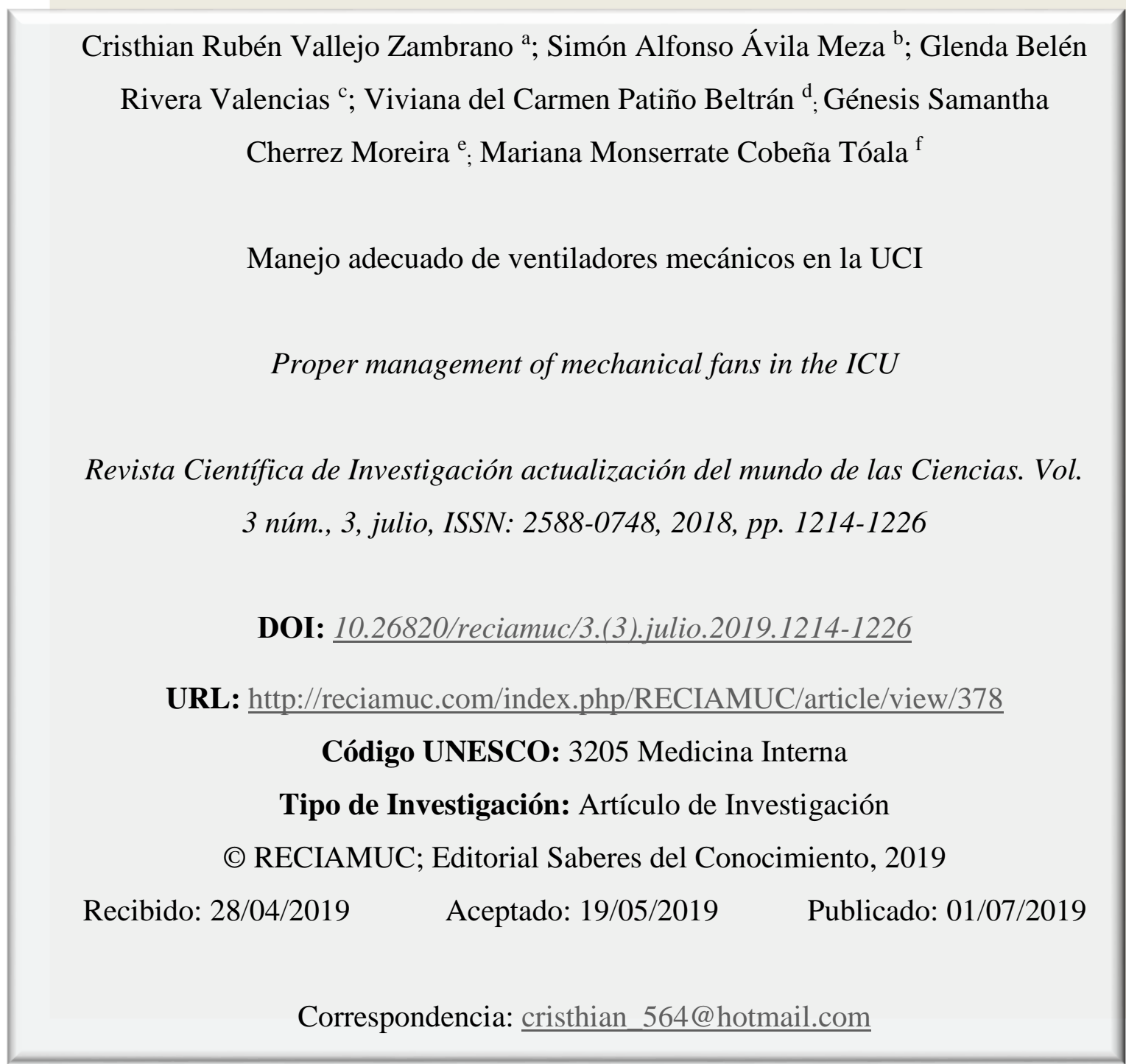

a. Médico Cirujano de 3er Nivel de Atención; Clínica Cardiocentro Manta - INCAP - CICCMA; cristhian564@hotmail.com; Manta - Ecuador.

b. Médico Cirujano; Clínica del Sol; simonalfonsoavilamezabsc@ hotmail.com; Manta - Ecuador.

c. Médico; Médico ocupacional - Soluciones Avanzadas Informáticas y Telecomunicaciones SAITEL; belegrv@hotmail.com; Ibarra- Ecuador.

d. Médico; vivi021911@ @otmail.com; Saraguro Ecuador.

e. Médico Cirujano; samychemor92@gmail.com; Portoviejo - Ecuador.

f. Médico Rural Centro de Salud Estero Ciego, Directora de la Unidad; mary_sab15@ @otmail.com; Pajan - Ecuador. 


\section{Manejo adecuado de ventiladores mecánicos en la UCI}

Vol. 3, núm. 3., (2019)

Cristhian Rubén Vallejo Zambrano; Simón Alfonso Ávila Meza; Glenda Belén Rivera

Valencias; Viviana del Carmen Patiño Beltrán; Génesis Samantha Cherrez Moreira; Mariana

Monserrate Cobeña Tóala

\section{RESUMEN}

Existen estados críticos en que las personas no pueden valerse por ellos mismos para realizar las funciones básicas del organismo, para los cuales los hospitales destinan un área especializada llamada Unidad de Cuidados Intensivos (UCI). Uno de los equipos más usados en esta área es el ventilador mecánico, que requiere un manejo adecuado para evitar complicaciones derivadas de su uso. El objetivo básico de esta revisión consiste en plasmar aspectos fundamentales relacionados con la ventilación mecánica usada por el paciente en la UCI. El diseño de investigación que se llevó a cabo es de tipo documental o bibliográfico. Los protocolos son indicaciones estándares fundamentadas en la evidencia que garantizan un buen manejo de los equipos de la UCI y buscan la estabilidad del paciente crítico. El debido uso de la ventilación mecánica garantiza la reducción de las tasas de neumonía y otras complicaciones graves que de ella se derivan. Se concluye que es fundamental la elaboración e implementación de protocolos que permitan la unificación de criterios con base en evidencia científica y la minimización de los riesgos asociados al uso de la ventilación mecánica en la UCI, asimismo, la constante especialización del personal que labora en dicha área.

Palabras Claves: Manejo; Adecuado; Ventilador; Mecánico; Crítico. 


\section{Manejo adecuado de ventiladores mecánicos en la UCI}

Vol. 3, núm. 3., (2019)

Cristhian Rubén Vallejo Zambrano; Simón Alfonso Ávila Meza; Glenda Belén Rivera

Valencias; Viviana del Carmen Patiño Beltrán; Génesis Samantha Cherrez Moreira; Mariana Monserrate Cobeña Tóala

\section{ABSTRACT}

There are critical states in which people cannot use themselves to perform the basic functions of the organism, for which hospitals allocate a specialized area called Intensive Care Unit (ICU). One of the most used equipment in this area is the mechanical fan, which requires proper handling to avoid complications arising from its use. The basic objective of this review is to capture fundamental aspects related to the mechanical ventilation used by the patient in the ICU. The research design that was carried out is documentary or bibliographic. The protocols are standard indications based on the evidence that guarantee a good management of the ICU equipment and seek the stability of the critical patient. The due use of mechanical ventilation guarantees the reduction of pneumonia rates and other serious complications that result from it. It is concluded that the development and implementation of protocols that allow the unification of criteria based on scientific evidence and the minimization of the risks associated with the use of mechanical ventilation in the ICU is essential, as well as the constant specialization of the personnel working in said area.

Key Words: Handling; Adequate; Fan; Mechanic; Critical. 


\section{Manejo adecuado de ventiladores mecánicos en la UCI}

Vol. 3, núm. 3., (2019)

Cristhian Rubén Vallejo Zambrano; Simón Alfonso Ávila Meza; Glenda Belén Rivera

Valencias; Viviana del Carmen Patiño Beltrán; Génesis Samantha Cherrez Moreira; Mariana

Monserrate Cobeña Tóala

\section{Introducción.}

El estado crítico de un paciente denota inestabilidad en los signos vitales y dependencia para llevar a cabo algunas funciones básicas del organismo para sobrevivir, estos pacientes requieren ayuda especializada, tanto de personal como de equipos médicos.

Estos expertos son los encargados de brindar cuidados especializados a los pacientes que presentan necesidades médicas críticas, los cuales requieren riguroso control y cuidados avanzados. "Todos los servicios de cuidados intensivos están a cargo de médicos y otros profesionales del área de la salud especialmente capacitados en cuidados intensivos (intensivistas)". (Mayo Clinic, 2018)

En este orden de ideas, la terapia intensiva es la encargada de brindar apoyo a pacientes en estado crítico, ya sea como consecuencia de una cirugía, enfermedad o accidente. La terapia intensiva se caracteriza por una atención médica cercana al paciente y constante, por parte de un equipo de profesionales de la salud que deben estar especialmente capacitados. Este servicio es brindado en las unidades de cuidados intensivos (UCI). En estas salas especializadas que brinda la ayuda necesaria al paciente en estado crítico se encuentran equipos especializados tales como monitores, ventiladores y otros equipos, vías intravenosas, sondas de alimentación, catéteres, entre otros. Son métodos y equipos cuyo propósito es mantener con vida a una persona, no obstante, pueden aumentar el riesgo de infecciones. (Biblioteca Nacional de Medicina de los Estados Unidos, 2019)

Sus objetivos fundamentales son la monitorización, por una parte, y el apoyo de las funciones vitales del paciente, aquellas que por sí solo el paciente en estado crítico no pueda controlar, todo ello con el propósito de estabilizarlo, efectuar diagnósticos y administrar tratamientos que permitan la evolución satisfactoria del mismo.

Una de las terapias más usadas en la UCI es la ventilación mecánica, la cual, según (Gutiérrez, 2011) se realiza mediante una "máquina que suministra un soporte ventilatorio y oxigenatorio, facilitamos el intercambio gaseoso y el trabajo respiratorio de los pacientes con insuficiencia respiratoria". (p. 87) 


\section{Manejo adecuado de ventiladores mecánicos en la UCI}

Vol. 3, núm. 3., (2019)

Cristhian Rubén Vallejo Zambrano; Simón Alfonso Ávila Meza; Glenda Belén Rivera

Valencias; Viviana del Carmen Patiño Beltrán; Génesis Samantha Cherrez Moreira; Mariana Monserrate Cobeña Tóala

Esta terapia permite el tratamiento con éxito de la insuficiencia respiratoria aguda (IRA), además de mantener con vida al paciente. En contraposición con sus valiosas ventajas esta terapia de ventilación se encuentra asociada a numerosas complicaciones, por tal razón resulta vital el buen manejo de estos equipos y el uso de las técnicas adecuadas. "Un mal manejo de la ventilación mecánica, no solo puede producir al paciente consecuencias graves sobre su sistema respiratorio, sino que también pueden ocasionarle graves trastornos hemodinámicos y como consecuencia de ello afectarse todo el organismo”. (Puga, y otros, 2014)

Dentro de sus principales complicaciones se pueden situar la neumonía y la dependencia de la ventilación artificial, siendo ambos peligrosos para el paciente de la UCI.

El objetivo básico de esta revisión consiste en plasmar aspectos fundamentales relacionados con la ventilación mecánica usada por el paciente en la UCI, conceptos, riesgos, protocolos y manejo adecuado del ventilador y técnicas asociadas al cuidado que del uso de este se derivan, en paciente crítico ingresado a la sala de cuidados intensivos.

\section{Materiales y Métodos.}

Se llevó a cabo una búsqueda de información organizada, en base a bibliografía relacionada sobre la ventilación mecánica usada por el paciente en la UCI. Se recopiló la información y se realizó el análisis de la literatura, asimismo se llevó a cabo la redacción de los resultados centrados en las siguientes preguntas: ¿Definición de Ventilación Mecánica?, ¿Riesgos que presenta el paciente en la UCI asistido por ventilación mecánica?, ¿Situación actual de los protocolos de prevención de neumonía asociada a ventiladores mecánicos? Y ¿Cuál es el manejo o cuáles son los cuidados específicos derivados de la Ventilación Mecánica?

Se realizó una revisión de la literatura a partir de las bases de datos, PubMed, MedLine y Web of Science, utilizando las expresiones o descriptores "Manejo del paciente con ventilación mecánica en UCI", "Ventilación Mecánica en UCI", "Aparatos utilizados en la Unidad de Cuidados Intensivos". 


\section{Manejo adecuado de ventiladores mecánicos en la UCI}

Vol. 3, núm. 3., (2019)

Cristhian Rubén Vallejo Zambrano; Simón Alfonso Ávila Meza; Glenda Belén Rivera Valencias; Viviana del Carmen Patiño Beltrán; Génesis Samantha Cherrez Moreira; Mariana Monserrate Cobeña Tóala

Luego de localizar los artículos de interés y páginas con información de salud certificadas como confiables, se filtraron bajo los criterios de idioma español, relevancia, correlación temática y fecha de publicación en los últimos diez años, sin descartar por tipo de material bibliográfico, quedando finalmente un total de 10 fuentes bibliográficas para el análisis.

\section{Resultados.}

\section{Ventilación Mecánica (VM).}

Consiste en un tratamiento de soporte vital. Un ventilador o respirador mecánico es una máquina que proporciona ayuda al paciente para respirar, e aquellos casos en que esta no es capaz por sí misma. "La mayoría de los pacientes que necesitan la ayuda de un ventilador debido a una enfermedad severa están internados en la unidad de cuidados intensivos (UCI) de un hospital". (American Thoracic Society, 2013)

Para Schwonke, Lunardi, \& Silva (2014) la ventilación mecánica puede ser clasificada en dos tipos: la ventilación mecánica invasiva y la ventilación mecánica no invasiva. Un aspecto en común es que las dos ventilaciones se dan por medio de presión positiva. La diferencia radica en la forma en que se libera esta presión. "En la primera se utiliza un tubo oro o nasotraqueal o una cánula de traqueotomía; en la VM no invasiva el dispositivo de conexión entre el paciente y el ventilador artificial consiste en una máscara". (p. 254)

\section{Ventilación Mecánica Invasiva.}

La ventilación mecánica invasiva se define como un mecanismo agresivo para el paciente pero que a su vez representa una serie de ventajas sobre todo en aquellos casos donde se estima que el mismo estará conectado a ventilación mecánica por un largo periodo de tiempo. Se refiere básicamente al tipo de mecanismo usado para ventilar al paciente, el cual puede ser por medio de un tubo introducido por la vía oro o nasotraqueal o una cánula de traqueotomía.

Ventilación mecánica no invasiva. 


\section{Manejo adecuado de ventiladores mecánicos en la UCI}

Vol. 3, núm. 3., (2019)

Cristhian Rubén Vallejo Zambrano; Simón Alfonso Ávila Meza; Glenda Belén Rivera

Valencias; Viviana del Carmen Patiño Beltrán; Génesis Samantha Cherrez Moreira; Mariana Monserrate Cobeña Tóala

Existe un mecanismo de ventilación menos agresivo, y por tanto con menos complicaciones, que brinda soporte para la respiración por medio de mascaras. Para Raurell et al. (2015) El objetivo básico de la ventilación mecánica no invasiva (VNI) es evitar la intubación traqueal, asimismo, facilita la extubación precoz y evita la reintubación, con todo esto disminuye el riesgo de neumonía que pueda asociarse a la ventilación mecánica, además de disminuir la estancia en la UCI, y por ende, disminuye también el gasto sanitario. (p. 47)

Riesgos de la ventilación mecánica.

\section{Infecciones.}

El tubo de traqueotomía facilita el ingreso de gérmenes a los pulmones. Como consecuencia el paciente puede presentar neumonía, que puede ser un grave problema que aumente la permanencia del uso del respirador mecánico. Asimismo, puede dañar los pulmones. Existen factores de riesgo controlables y otros no controlables para las neumonías asociadas a la ventilación mecánica, entre los no controlables están las personas de mayor edad y los pacientes muy enfermos. (American Thoracic Society, 2013)

\section{Colapso pulmonar (neumotórax).}

Se produce cuando se filtra el aire dentro del espacio que se encuentra entre los pulmones y la pared torácica. "Puede ser colapso pulmonar completo o solo una parte del pulmón. Se puede producir un tipo grave de neumotórax en personas que necesitan asistencia mecánica para respirar. El respirador puede crear un desequilibrio de presión de aire dentro del pecho". (Mayo Clinic, 2019)

Una vez producido el neumotórax, se debe extraer el aire de esta cavidad para liberar la presión del pulmón. El médico puede colocar un tubo distinto en el tórax entre las costillas con la finalidad de drenar el exceso de aire. Esta acción permite que el pulmón se vuelva a expandir y selle la pérdida. "Generalmente, se debe dejar colocado el tubo torácico un tiempo para asegurarse de que se haya detenido la pérdida y extraer todo el aire. En casos infrecuentes, el colapso repentino de un pulmón puede causar la muerte”. (American Thoracic Society, 2013) 


\section{Manejo adecuado de ventiladores mecánicos en la UCI}

Vol. 3, núm. 3., (2019)

Cristhian Rubén Vallejo Zambrano; Simón Alfonso Ávila Meza; Glenda Belén Rivera

Valencias; Viviana del Carmen Patiño Beltrán; Génesis Samantha Cherrez Moreira; Mariana

Monserrate Cobeña Tóala

\section{Daño pulmonar.}

$\mathrm{Al}$ colocar aire dentro de los pulmones con un ventilador, esta presión puede dañarlos. Este riesgo se trata de minimizar con la aplicación de menos cantidad de presión posible. Los altos niveles de oxígeno también resultan dañinos para los pulmones. Una complicación grave que impide minimizar este riesgo es cuando ya los pulmones están dañados. (American Thoracic Society, 2013)

Mantenimiento de la vida.

Cuando el paciente presenta una enfermedad muy avanzada el uso del ventilador mecánico de la única menara que influye es en posponer la muerte. Su uso no denota en este caso una mejoría del paciente. No obstante, algunas veces, los médicos tienen confianza en que el uso del ventilador ayudará en la recuperación del paciente. En otras ocasiones, los médicos no podrán asegurar que el paciente sobreviva. En muchos casos se presenta la posibilidad de preguntar al paciente o a un familiar cercano, si se mantiene el respirador, sólo en aquellos casos en que no presente mejorías o su estado empeore. (American Thoracic Society, 2013)

\section{Protocolos.}

Uno de los principales problemas en materia de organización de salud a nivel mundial es la unificación de criterios. La elaboración de guías para el adecuado manejo de los equipos y procedimientos adecuados en las salas de cuidados intensivos no son la excepción, en todo el mundo existe un sinfín de guías y protocolos acerca de esta temática y algunos estudios coinciden en que el éxito de la atención en la UCI está dada por la unidad de criterios en base a evidencias sólidas, materializada en la existencia de protocolos precisos.

La capacitación constante por parte del equipo de salud que trabaja en la sala de cuidados intensivos es fundamental, especialmente el personal de enfermería sobre el cual recae la mayor atención y cuidado de los pacientes ingresados en esta unidad. 


\section{Manejo adecuado de ventiladores mecánicos en la UCI}

Vol. 3, núm. 3., (2019)

Cristhian Rubén Vallejo Zambrano; Simón Alfonso Ávila Meza; Glenda Belén Rivera

Valencias; Viviana del Carmen Patiño Beltrán; Génesis Samantha Cherrez Moreira; Mariana Monserrate Cobeña Tóala

Un buen protocolo de atención en la UCI y un personal calificado van a ser bases sólidas para una buena atención y estabilización del paciente crítico, así como la minimización de las complicaciones que puedan presentarse consecuencia de los procedimientos llevados a cabo en esta área.

Una de las principales complicaciones del paciente ingresado a UCI es la Neumonía Asociada al uso de Ventilador Mecánico (NAVM), no obstante, el buen manejo de los equipos de la UCI, especialmente del ventilador y el buen cuidado pueden minimizar los riesgos de que esta afección se presente y empeore el estado del paciente.

Villamón Nevot (2015) en los datos recogidos en su estudio, demuestran una disminución de la tasa de incidencia de Neumonía Asociada a Ventilador Mecánico (NAVM) tras la implantación del protocolo de prevención del proyecto neumonía zero de España, realizado sobre una “elección de componentes se basa en la evidencia actual que aportan diferentes organismos como la clasificación GRADE, Task Force Canadiense, las recomendaciones del Centers for Disease Control and Prevention (CDC) o las que realiza el Instituto Joanna Briggs", estas se compendiaron en las siguientes indicaciones: 1. Formación del personal: resaltando la manipulación de vía aérea. 2. Lavado de manos y guantes, es importante cuando se está en contacto con secreciones y equipos de ventilación. 3. Intubación orotraqueal mejor que la nasotraqueal. 4. Favorecer todos los procedimientos que permitan disminuir de forma segura la intubación y/o su duración. 5. Evitar la posición de decúbito supino. 6. Presión óptima de neumotaponamiento entre 20-30 cm H2O. 7. Evitar las secreciones que se acumulan por encima del tubo endotraqueal. 8. Humidificadores e intercambiadores de calor-humedad. 9. Se recomienda la desinfección con antiséptico. 10. Descontaminación selectiva digestiva. (p. 104, 105)

La implantación de buenos protocolos sobre evidencia científica garantiza mejores resultados y minimizan las complicaciones que puedan presentarse en esta área.

Manejo o cuidados específicos derivados de la Ventilación Mecánica.

En el momento de intubar. 


\section{Manejo adecuado de ventiladores mecánicos en la UCI}

Vol. 3, núm. 3., (2019)

Cristhian Rubén Vallejo Zambrano; Simón Alfonso Ávila Meza; Glenda Belén Rivera Valencias; Viviana del Carmen Patiño Beltrán; Génesis Samantha Cherrez Moreira; Mariana Monserrate Cobeña Tóala

Para Armes, Mosegue, \& Gallowey (2014) La intubación es un procedimiento que requiere destreza, rapidez y coordinación por cada uno de los miembros. Para la intubación se debe considerar en primer lugar la preparación del material a utilizar (set de intubación). Se debe comprobar la luz del laringoscopio y la impermeabilidad del balón. "El paciente debe estar correctamente sedado, relajado y analgesicado. El ventilador enchufado con parámetros prefijados: FiO2, modalidad, FR, Vc y tipo de flujo. El paciente debe estar en todo momento con monitorización cardiaca y pulsioxímetro". (p. 23)

\section{De la vía aérea artificial (cuidados de la intubación).}

El cuidado de la intubación o aspiración de secreciones es fundamental. Es una labor que debe ser realizada bajo la máxima asepsia y generando el mínimo traumatismo. En cada aspiración no deben ser empleados más de $15 \mathrm{sg}$. Se debe tratar de hacer vacío solamente durante la retirada del catéter. En ocasiones se requiere lubricar la sonda de aspiración con la finalidad de hacer más fácil la penetración. Es conveniente la administración de $\mathrm{O} 2$ al 100\% antes de una aspiración, en pacientes con mayor inestabilidad. Con respecto a la humidificación, calentamiento y filtración del aire inspirado, es importante tener en cuenta que los filtros intercalados en el circuito inhiben el crecimiento de bacterias y hongos. El sistema de cascada presenta ventajas sobre el llamado de nariz artificial, ya que no sólo humidifica, sino que también calienta el aire. Por último, con relación a la colocación y mantenimiento óptimo del tubo, se puede aplicar una regla mnemotécnica que ayuda o es una guía para la correcta posición del tubo, esta es la regla de las tres "T" (Tube, Theeth y Twenty two), que se refiere a que el tubo suele estar apoyado sobre los

dientes en su número 22. Asimismo, es importante mantener y comprobar la presión del neumotaponamiento y evitar erosiones externas. La hidratación de los labios y una buena higiene bucal son importantes. (Armes, Mosegue, \& Gallowey, 2014, p. 23)

\section{Cuidados de la vía aérea artificial (cuidados de la traqueotomía).}

La traqueotomía es la "apertura de una vía en la cara anterior de la tráquea con el objeto de permeabilizar la vía aérea mediante la introducción de una cánula". Es recomendable sobre todo en aquellos casos de pacientes que van a permanecer intubados un largo tiempo. En estos casos el 


\section{Manejo adecuado de ventiladores mecánicos en la UCI}

Vol. 3, núm. 3., (2019)

Cristhian Rubén Vallejo Zambrano; Simón Alfonso Ávila Meza; Glenda Belén Rivera

Valencias; Viviana del Carmen Patiño Beltrán; Génesis Samantha Cherrez Moreira; Mariana Monserrate Cobeña Tóala

riesgo de lesión en la mucosa traqueal es menor, otra ventaja es que la cánula se desplaza menos y la aspiración resulta más fácil, así como la higiene bucal se puede realizar mejor, en líneas generales es más cómodo para el paciente. En estos casos es importante evitar desplazamientos bruscos. Cuidar la estoma debidamente, realizar los cambios de cánula. En general, van orientados a cubrir fundamentalmente las necesidades del paciente crítico de oxigenación, eliminaciónnutrientes y seguridad y bienestar. (Armes, Mosegue, \& Gallowey, 2014, p. 23,24)

\section{Conclusión.}

Los protocolos para el buen uso de los equipos de la UCI son una herramienta fundamental en la organización de esta área especializada. La prevención de Neumonía asociada al uso de ventilación mecánica puede ser reducida gracias a la implantación de estos protocolos. Es importante en cada procedimiento médico o del equipo de salud encargado del cuidado de un paciente, la unificación de criterios y la elaboración y puesta en práctica de guías que les permitan seguir un máximo estándar de calidad, apoyado en evidencia científica con la finalidad de brindar la mejor atención y proporcionar el mejor cuidado al paciente para una mayor y más rápida evolución.

La ventilación mecánica es una herramienta que facilita la estabilidad del paciente crítico con problemas de respiración, no obstante, se requiere un cuidado especializado, adecuado, que permita minimizar las complicaciones que del uso de estos aparatos y técnicas asociadas se derivan y evitar males mayores.

En este orden de ideas, es imperioso la constante actualización del personal de la UCI en el manejo de los equipos, así como la revisión y la actualización de las guías o protocolos. En un mundo donde la tecnología pone a la mano herramientas más eficaces cada día, es necesario ir a la par con la actualización de estas herramientas, que requieren nuevos procedimientos y personal debidamente entrenando.

En la actualidad, los ventiladores mecánicos se han convertido en respiradores inteligentes, en donde la propia máquina verifica la evolución del paciente, así como la realización de los ajustes necesarios, con la finalidad de beneficiar la evolución del paciente, no obstante, la programación 


\section{Manejo adecuado de ventiladores mecánicos en la UCI}

Vol. 3, núm. 3., (2019)

Cristhian Rubén Vallejo Zambrano; Simón Alfonso Ávila Meza; Glenda Belén Rivera Valencias; Viviana del Carmen Patiño Beltrán; Génesis Samantha Cherrez Moreira; Mariana Monserrate Cobeña Tóala

de los equipos, la colocación de las vías y su manejo y control siguen en las manos del personal de UCI quienes deben estar a la altura de las exigencias tecnológicas en favor de brindar un mejor servicio.

La revisión realizada permitió identificar en la literatura los conceptos básicos de la ventilación mecánica, así como los principales riesgos de su uso con la finalidad de explicar posteriormente la importancia del uso de los protocolos de minimización de riesgos y los cuidados adecuados que del uso de este instrumento especializado se derivan.

\section{Bibliografía.}

American Thoracic Society. (Septiembre de 2013). thoracic.org/. Recuperado el 13 de Agosto de 2019, de https://www.thoracic.org/patients/patientresources/resources/spanish/mechanical-ventilation.pdf

Armes, A., Mosegue, R., \& Gallowey, M. (2014). Infomed Especialidades. (E. e. Intensivos, Ed.) Recuperado el 11 de Agosto de 2019, de https://especialidades.sld.cu/enfermeriaintensiva/files/2014/04/vent_mecanic_princ_basic .pdf

Biblioteca Nacional de Medicina de los Estados Unidos. (25 de Julio de 2019). MedlinePlus. Recuperado el 12 de Agosto de 2019, de https://medlineplus.gov/spanish/criticalcare.html

Gutiérrez, F. (2011). Ventilación Mecánica. Acta Med Per, 28(287-104). Recuperado el 10 de Agosto de 2019, de http://www.scielo.org.pe/pdf/amp/v28n2/a06v28n2.pdf

Mayo Clinic. (29 de Noviembre de 2018). mayoclinic.org/es. Recuperado el 13 de Agosto de 2019, de https://www.mayoclinic.org/es-es/departments-centers/criticalcare/sections/overview/ovc-20399554

Mayo Clinic. (20 de Junio de 2019). mayoclinic.org. Recuperado el 13 de Agosto de 2019, de https://www.mayoclinic.org/es-es/diseases-conditions/pneumothorax/symptomscauses/syc-20350367

Puga, M., Parellada, J., Hernández, W., Pérez, F., Quiñónez, A., \& Ruíz, L. (2014). Propuesta de algoritmos para la ventilación mecánica en las áreas intensivas municipales y los centros de diagnóstico integral. Revista Cubana de Medicina Intensiva y Emergencia, 13(1). $\begin{array}{lllllll}\text { Recuperado el } 12 \text { de Agosto de } & \text { 2019, }\end{array}$ http://www.revmie.sld.cu/index.php/mie/article/view/10/36 


\section{Manejo adecuado de ventiladores mecánicos en la UCI}

Vol. 3, núm. 3., (2019)

Cristhian Rubén Vallejo Zambrano; Simón Alfonso Ávila Meza; Glenda Belén Rivera

Valencias; Viviana del Carmen Patiño Beltrán; Génesis Samantha Cherrez Moreira; Mariana

Monserrate Cobeña Tóala

Raurell, M.; Argilaga, E.; Colomer, M.; Ruiz, T.; Galvany, A.; González, A. (02 de Abril de 2015). Análisis comparativo de los conocimientos en ventilación mecánicano invasiva de profesionales de cuidados intensivos, 26(2), 46-53. Recuperado el 10 de Agosto de 2019, de https://www.elsevier.es/es-revista-enfermeria-intensiva-142-pdf-S1130239915000309

Schwonke, C., Lunardi, W., \& Silva, J. (2014). Ambiente y Ventilación Mecánica: Una reflexión posible. Enfermería Global(35), 254-262. Recuperado el 14 de Agosto de 2019, de http://scielo.isciii.es/pdf/eg/v13n35/reflexion2.pdf

Villamón Nevot, M. J. (Abril de 2015). Evaluación del cumplimiento de un protocolo de prevención de Neumonía asociada a Ventilación mecánica en una UCI polivalente. Enefremería Global(38), 102-117. Recuperado el 12 de Agosto de 2019, de http://scielo.isciii.es/pdf/eg/v14n38/clinica5.pdf

$$
\text { (9) }(\mathcal{O Q})
$$

RECONOCIMIENTO-NOCOMERCIAL-COMPARTIRIGUAL

CC BY-NC-SA

ESTA LICENCIA PERMITE A OTROS ENTREMEZCLAR, AJUSTAR Y CONSTRUIR A PARTIR DE SU OBRA CON FINES NO

COMERCIALES, SIEMPRE Y CUANDO LE RECONOZCAN LA AUTORÍA Y SUS NUEVAS CREACIONES ESTÉN BAJO UNA LICENCIA CON LOS MISMOS TÉRMINOS. 